\title{
Radio Tomographic Imaging with Wireless Networks
}

\author{
Joey Wilson and Neal Patwari \\ The University of Utah
}

\begin{abstract}
Radio Tomographic Imaging (RTI) is an emerging technology for imaging the attenuation caused by physical objects in wireless networks. This paper presents a linear model for using received signal strength (RSS) measurements to obtain images of moving objects. Noise models are investigated based on real measurements of a deployed RTI system. Mean-squared error (MSE) bounds on image accuracy are derived, which are used to calculate the accuracy of an RTI system for a given node geometry. The ill-posedness of RTI is discussed, and Tikhonov regularization is used to derive an image estimator. Experimental results of an RTI experiment with 28 nodes deployed around a 441 square foot area are presented.
\end{abstract}

Index Terms-Wireless, Sensor Networks, Inverse Filtering, Linear Systems, Applications

\section{INTRODUCTION}

W HEN an object moves into the area of a wireless network, links which pass through that object will experience shadowing losses. This paper explores in detail the use of shadowing losses on links between many pairs of nodes in a wireless network to image the attenuation of objects within the network area. We refer to this problem as radio tomographic imaging (RTI), as depicted in Fig. 1.

RTI may be useful in emergencies, rescue operations, and security breaches, since the objects being imaged need not carry an electronic device. Using the images to track humans moving through a building, for example, provides a basis for new applications in security systems and "smart" buildings.

The reduction in costs for radio frequency integrated circuits (RFICs) and advances in peer-to-peer data networking have made realistic the use of hundreds or thousands of simple radio devices in a single RTI deployment. Since the relative cost of such devices is low, large RTI networks are possible in applications that may be otherwise impractical.

Radio tomography draws from the concepts of two well-known and widely used types of imaging systems. First, radar systems transmit RF probes and receive echoes caused by the objects in an environment [1]. A delay between transmission and reception indicates a distance to a scatterer. Phased array radars also compute an angle of bearing. Such systems image an object in space based on reflection and scattering. Secondly, computed tomography (CT) methods in medical and geophysical imaging systems use signal measurements along many different paths through a medium. The measurements along the paths are used to compute an estimate of the spatial field of the transmission parameters throughout the medium [2]. RTI is also a transmission-based imag-

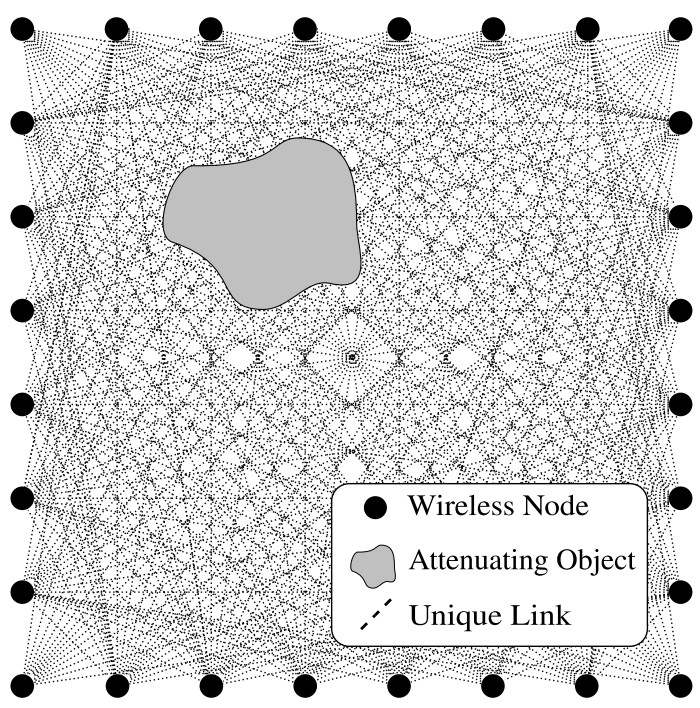

Fig. 1. An illustration of an RTI network. Each node broadcasts to the others, creating many projections that can be used to reconstruct an image of objects inside the network area.

ing method which measures signal strengths on many different paths through a medium, but similar to radar systems, it does so at radio frequencies. It also faces two significant challenges:

- The system discussed in this paper measures only signal strength. No information about the phase or the time-domain of a signal is available.

- The use of RF, as opposed to much higher frequency EM waves (e.g., x-rays), introduces significant nonline-of-sight (NLOS) propagation in the transmission measurements. Signals in standard commercial wireless bands do not travel in just the line-of-sight (LOS) path, and instead propagate in many paths from a transmitter to a receiver. 


\subsection{Applications}

Despite the difficulties of using RF, there is a major advantage: RF signals can travel through obstructions such as walls, trees, and smoke, while optical or infrared imaging systems cannot. RF imaging will also work in the dark, where video cameras will fail. Even for applications where video cameras could work, privacy concerns may prevent their deployment. An RTI system provides current images of the location of people and their movements, but cannot be used to identify a person.

One main future application of RTI is to reduce injury for correctional and law enforcement officers; many are injured each year because they lack the ability to detect and track offenders through building walls [3]. By showing the locations of people within a building during hostage situations, building fires, or other emergencies, RTI can help law enforcement and emergency responders to know where they should focus their attention.

Another application is in automatic monitoring and control in "smart" homes and buildings. Some building control systems detect motion in a room and use it to control lighting, heating, air conditioning, and even noise cancellation [4]. RTI systems can further determine how many people are in a room and where they are located, providing more precise control.

RTI has application in security and monitoring systems for indoor and outdoor areas. For example, most existing security systems are trip-wire based or camerabased. Trip-wire systems detect when people cross a boundary, but do not track them when they are within the area. Cameras are ineffective in the dark and have limited view angles. An RTI system could serve both as a trip-wire, alerting when intruders enter into an area, and a tracking system to follow their movements.

\subsection{Related Work}

RF-based imaging has been dominated in the commercial realm by ultra-wideband (UWB) based through-thewall (TTW) imaging devices from companies like Time Domain [5], Cambridge Consultants [6], and Camero Tech [7]. These companies have developed products using a phased array of radars that transmit UWB pulses and then measure echoes to estimate a range and bearing. These devices are accurate close to the device, but inherently suffer from accuracy and noise issues at long range due to mono-static radar scattering losses and large bandwidths. Some initial attempts [8] allow 2-4 of these high-complexity devices to collaborate to improve coverage.

In comparison, in this paper we discuss using dozens to hundreds of low-capability collaborating nodes, which measure transmission rather than scattering and reflection. Further, UWB uses an extremely wide RF bandwidth, which may limit its application to emergency and military applications. RTI is capable of using radios with relatively small bandwidths.
To emphasize the small required bandwidth compared to UWB, some relevant research is being called "ultranarrowband" (UNB) radar [9], [10], [11]. These systems propose using narrowband transmitters and receivers deployed around an area to image the environment within that area. Measurements are phase-synchronous at the multiple nodes around the area. Such techniques have been applied to detect and locate objects buried under ground using what is effectively a synthetic aperture array of ground-penetrating radars [12]. Experiments have been reported which measure a static environment while moving one transmitter or one receiver [11], and measure a static object on a rotating table in an anechoic chamber in order to simulate an array of transmitters and receivers at many different angles [11], [12], [9].

Multiple-input-multiple-output (MIMO) radar is another emerging field that takes advantage of multiple transmitters and receivers to locate objects within a spatial area [13]. In this framework, signals are transmitted into the area of interest, objects scatter the signal, and the reflections are measured at each receiver. The scattering objects create a channel matrix which is comparable to the channel matrix in MIMO communication theory. RTI differs from MIMO radar in the same way that it differs from traditional radar. Instead of measuring reflections, RTI uses the shadowing caused by objects as a basis for image reconstruction.

Recent research has also used measurements of signal strength on $802.11 \mathrm{WiFi}$ links to detect and locate a person's location. Experiments in [14] demonstrate the capability of a detector based on signal strength measurements determine the location of a person who is not carrying an electronic device. In this case, the system is trained by a person standing at pre-defined positions, and RSS measurements are recorded at each location. When the system is in use, RSS measurements are compared with the known training data, and the best position is selected from a list.

Our approach is not based on point-wise detection. Instead, we use tomographic methods to estimate an image of the change in the attenuation as a function of space, and use the image estimate for the purposes of indicating the position of a moving object.

\subsection{Overview}

Section 2 presents a linear model relating RSS measurements to the change in attenuation occurring in a network area, and investigates statistics for noise in dynamic multipath environments. Section 3 describes an error bound on image estimation for a given node geometry. This is useful to determine which areas of a network can be accurately imaged for a given set of node locations. Section 4 discusses the ill-posedness of RTI, and derives a regularized solution for obtaining an attenuation image. Section 5 describes the setup of an actual RTI experiment, the resultant images, and a discussion of the effect of parameters on the accuracy of the images. 


\section{MOdeL}

\subsection{Linear Formulation}

When wireless nodes communicate, the radio signals pass through the physical area of the network. Objects within the area absorb, reflect, diffract, or scatter some of the transmitted power. The goal of an RTI system is to determine an image vector of dimension $\mathbb{R}^{N}$ that describes the amount of radio power attenuation occurring due to physical objects within $N$ voxels of a network region. Since voxel locations are known, RTI allows one to know where attenuation in a network is occurring, and therefore, where objects are located.

If $K$ is the number of nodes in the RTI network, then the total number of unique two-way links is $M=\frac{K^{2}-K}{2}$. Any pair of nodes is counted as a link, whether or not communication actually occurs between them. The signal strength $y_{i}(t)$ of a particular link $i$ at time $t$ is dependent on:

- $P_{i}$ : Transmitted power in $\mathrm{dB}$.

- $S_{i}(t)$ : Shadowing loss in $\mathrm{dB}$ due to objects that attenuate the signal.

- $F_{i}(t)$ : Fading loss in $\mathrm{dB}$ that occurs from constructive and destructive interference of narrow-band signals in multipath environments.

- $L_{i}$ : Static losses in $\mathrm{dB}$ due to distance, antenna patterns, device inconsistencies, etc.

- $\nu_{i}(t)$ : Measurement noise.

Mathematically, the received signal strength is described as

$$
y_{i}(t)=P_{i}-L_{i}-S_{i}(t)-F_{i}(t)-\nu_{i}(t)
$$

The shadowing loss $S_{i}(t)$ can be approximated as a sum of attenuation that occurs in each voxel. Since the contribution of each voxel to the attenuation of a link is different for each link, a weighting is applied. Mathematically, this is described for a single link as

$$
S_{i}(t)=\sum_{j=1}^{N} w_{i j} x_{j}(t) .
$$

where $x_{j}(t)$ is the attenuation occurring in voxel $j$ at time $t$, and $w_{i j}$ is the weighting of pixel $j$ for link $i$. If a link does not "cross" a particular voxel, that voxel is removed by using a weight of zero. For example, Fig. 2 is an illustration of how a direct LOS link might be weighted in a non-scattering environment.

Imaging only the changing attenuation greatly simplifies the problem, since all static losses can be removed over time. The change in RSS $\triangle y_{i}$ from time $t_{a}$ to $t_{b}$ is

$$
\begin{aligned}
\triangle y_{i} \equiv & y_{i}\left(t_{b}\right)-y_{i}\left(t_{a}\right) \\
= & S_{i}\left(t_{b}\right)-S_{i}\left(t_{a}\right)+F_{i}\left(t_{b}\right)-F_{i}\left(t_{a}\right) \\
& +\nu_{i}\left(t_{b}\right)-\nu_{i}\left(t_{a}\right),
\end{aligned}
$$

which can be written as

$$
\triangle y_{i}=\sum_{j=1}^{N} w_{i j} \triangle x_{j}+n_{i},
$$

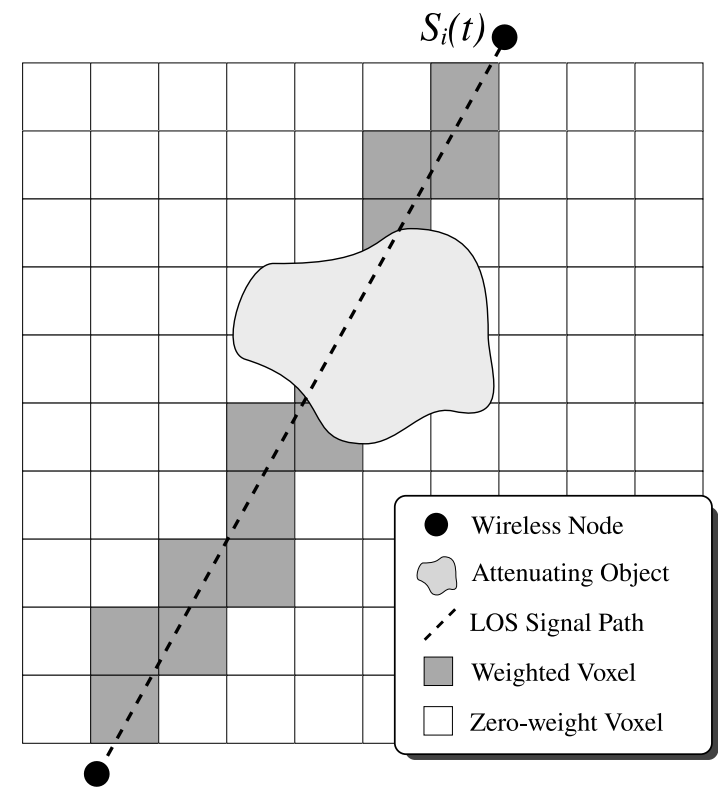

Fig. 2. An illustration of a single link in an RTI network that travels in a direct LOS path. The signal is shadowed by objects as it crosses the area of the network in a particular path. The darkened voxels represent the image areas that have a non-zero weighting for this particular link.

where the noise is the grouping of fading and measurement noise

$$
n_{i}=F_{i}\left(t_{b}\right)-F_{i}\left(t_{a}\right)+\nu_{i}\left(t_{b}\right)-\nu_{i}\left(t_{a}\right)
$$

and

$$
\triangle x_{j}=x_{j}\left(t_{b}\right)-x_{j}\left(t_{a}\right)
$$

is the difference in attenuation at pixel $j$ from time $t a$ to $t_{b}$.

If all links in the network are considered simultaneously, the system of RSS equations can be described in matrix form as

$$
\triangle \mathbf{y}=\mathbf{W} \triangle \mathbf{x}+\mathbf{n}
$$

where

$$
\begin{aligned}
\triangle \mathbf{y} & =\left[\triangle y_{1}, \triangle y_{2}, \ldots, \triangle y_{M}\right]^{T} \\
\triangle \mathbf{x} & =\left[\triangle x_{1}, \triangle x_{2}, \ldots, \triangle x_{N}\right]^{T} \\
\mathbf{n} & =\left[n_{1}, n_{2}, \ldots, n_{M}\right]^{T} \\
{[\mathbf{W}]_{i, j} } & =w_{i j}
\end{aligned}
$$

In summary, $\triangle \mathbf{y}$ is the vector of length $M$ all link difference RSS measurements, $\mathbf{n}$ is a noise vector, and $\triangle \mathbf{x}$ is the attenuation image to be estimated. $\mathbf{W}$ is the weighting matrix of dimension $M \times N$, with each column representing a single voxel, and each row describing the weighting of each voxel for that particular link. Each variable is measured in decibels $(\mathrm{dB})$.

To simplify the notation used throughout the rest of this paper, $\mathbf{x}$ and $\mathbf{y}$ are used in place of $\triangle \mathbf{x}$ and $\triangle \mathbf{y}$, respectively. 


\subsection{Weight Model}

If knowledge of an environment were available, one could estimate the weights $\left\{w_{i j}\right\}_{j}$ for link $i$ which reflected the spatial extent of multiple paths between transmitter and receiver. Perhaps calibration measurements could aid in estimation of the linear transformation $\mathbf{W}$. However, with no site-specific information, we require a statistical model that describes the linear effect of the attenuation field on the path loss for each link.

An ellipsoid with foci at each node location can be used as a method for determining the weighting for each link in the network [15]. If a particular voxel falls outside the ellipsoid, the weighting for that voxel is set to zero. If a particular voxel is within the LOS path determined by the ellipsoid, its weight is set to be inversely proportional to the square root of the link distance. Intuitively, longer links will provide less information about the attenuation in voxels that they cross. When link distances are very long, the signals reflect and defract around the obstructions. A link with a distance of only a few feet will experience more change in RSS when an obstruction occurs than a link with a length of hundreds of feet.

Past studies have shown that the variance of link shadowing does not change with distance. In accordance with these studies, dividing by the square root of the link distance ensures that the voxel weighting takes this into account [16]. The weighting is described mathematically as

$$
w_{i j}=\frac{1}{\sqrt{d}}\left\{\begin{array}{cc}
1 & \text { if } d_{i j}(1)+d_{i j}(2)<d+\lambda \\
0 & \text { otherwise }
\end{array}\right.
$$

where $d$ is the distance between the two nodes, $d_{i j}(1)$ and $d_{i j}(2)$ are the distances from the center of voxel $j$ to the two node locations for link $i$, and $\lambda$ is a tunable parameter describing the width of the ellipse.

The width parameter $\lambda$ is typically set very low in RTI, such that it is essentially the same as using the LOS model as depicted in Fig. 2. The use of an ellipsoid is primarily used to simplify the process of determining which voxels fall along the LOS path.

\subsection{Noise}

To complete the model of (7), the statistics of the noise vector $\mathbf{n}$ in (7) must be examined. Here, noise is caused by time-varying measurement miscalibration of the receiver, by the contribution of thermal noise to the measured receiver signal strength, and time-variations in the multipath channel not caused by changes to the attenuation experienced by the line-of-sight path. If these contributions are constant with time, then the calibration (when moving attenuator existed in the field) would have been able to establish it as the baseline. Time variation in RSS measurement when no moving attenuator is blocking the line-of-sight path is "noise" for an RTI system.

Past studies have considered the time-variation of RSS in fixed radio links. In particular, the work and measurements of Bultitude [17] were used to design indoor fixed wireless communications systems which periodically experienced fading due to motion in the area of the link. Bultitude found that RSS experienced intervals of significant fading which were caused by human motion in and around the area. Most of the time, the measured RSS vary slowly around a nearly constant mean, what we call a non-fading interval. When in a fading interval, RSS varies up to $10 \mathrm{~dB}$ higher and $20 \mathrm{~dB}$ below the non-fading interval mean, with a distribution that can be characterized as a Rician distribution [17]. Other measurements find temporal fading statistics more closely match a log-normal distribution [18]. The fading / non-fading interval process can be modeled as a twostate Markov chain [19], which alternates between a lowvariance and high-variance distribution. Over all time, measurements show a two-part mixture distribution for the RSS on a fixed link.

In linear terms, we could model this data as a mixture of two Rician distributions as in [17]; we could also model it as a mixture of log-normal terms as suggested by results in [18]. We note that the logarithm of a Rician random variable is often similar in distribution to the log-normal, perhaps a cause of disagreement between measurement studies. We choose to use the log-normal mixture model for simplicity; in the $(\mathrm{dB})$ scale, this is a two-part Gaussian mixture model:

$$
f_{n_{i}}(u)=\sum_{k \in\{1,2\}} \frac{p_{k}}{\sqrt{2 \pi \sigma_{k}^{2}}} \exp \left[-\frac{u^{2}}{2 \sigma_{k}^{2}}\right],
$$

where $p_{k}$ is the probability of part $k, p_{2}=1-p_{1}, \sigma_{k}^{2}$ is the variance of part $k$, and $f_{n_{i}}(u)$ is the probability density function of the noise random variable $n_{i}$. Without loss of generality, we let $\sigma_{2}>\sigma_{1}$ so that part 2 is the higher variance component of the mixture.

Past radio link measurements have not distinguished between motion which shadows the line-of-sight path (the signal in RTI), and motion which does not shadow the line-of-sight path (the noise) [17], [18], [19], [14]. To investigate the statistics of RTI noise, we present experimental tests which measure the time-varying statistics of links during motion which does not obstruct a link.

To collect experimental samples of noise, we set up 28 nodes in an indoor office area empty of people. While the nodes are transmitting and measuring RSS on each pairwise link, people move around the outside of the perimeter of the deployment area. In no case did the motion of a person obstruct the LOS path of any link. From each link, about 66,000 measurements were taken. For example, consider the data on a typical link, the link $(3,20)$. The temporal fading plot in Figure 3(a) shows similar results to [17], with alternating periods of heavy fading and low fading. During low fading, data is confined within a range of $2-3 \mathrm{~dB}$ around $-84 \mathrm{dBm}$. During high fading, variations at $\pm 10 \mathrm{~dB}$ from the mean occur. The histogram shown in Fig. 3(b) correspondingly shows a mixture of one high-variance and one lowvariance distribution. 


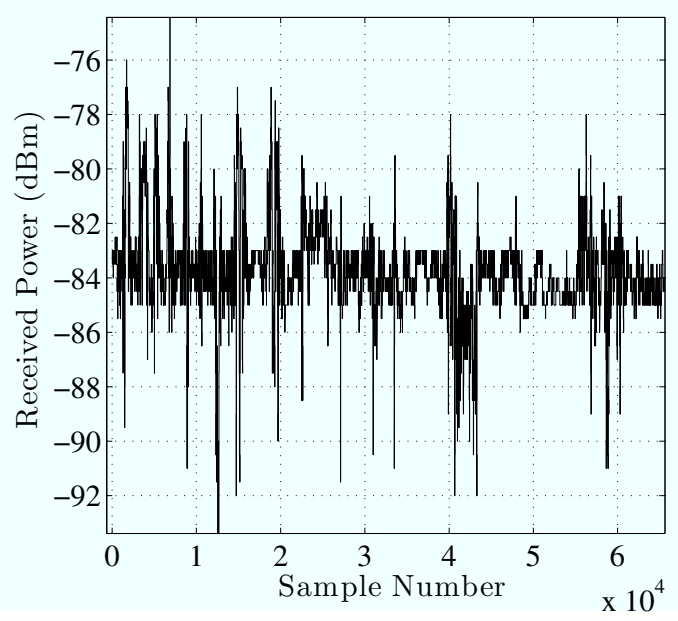

(a) Time plot

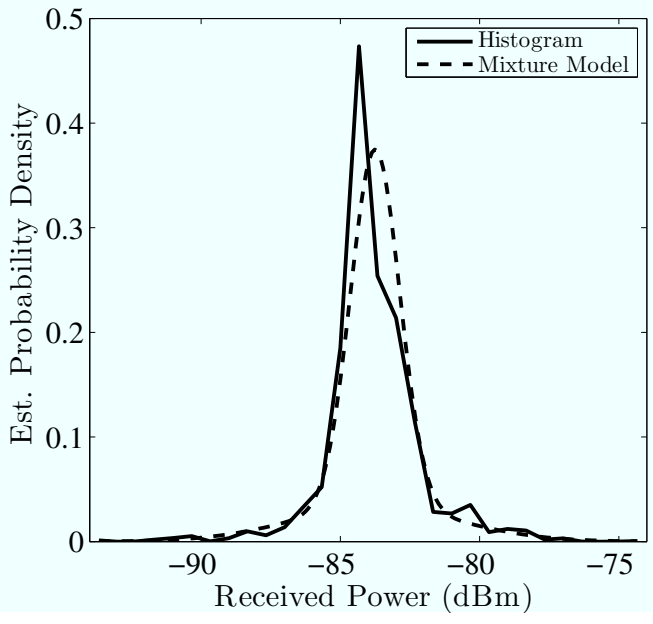

(b) Histogram

Fig. 3. Temporal fading on link $(3,20)$ during non-obstructing motion, showing (a) time plot and (b) histogram.

We also summarize the measured data on all $\left(\begin{array}{c}28 \\ 2\end{array}\right)$ links. The mean was removed from each link's data, and the data was merged. Fig. 4(a) is a quantile-quantile plot comparing the removed-mean RSS measurements with a Gaussian distribution $\mathcal{N}\left(0, \sigma_{d}^{2}\right)$, where $\sigma_{d}^{2}$ is the empirical variance of the measurements. The PDF is approximated by a Gaussian within \pm 2.5 quantiles.

As described, the data seems to follow a mixture distribution. From measured data, we estimate the mixture parameters with an expectation-maximization (EM) algorithm [20], and the results are shown in Table 1. Fig. $4(\mathrm{~b})$ is a quantile-quantile plot comparing the removedmean RSS measurements with a mixture model with the stated parameters.

\begin{tabular}{|c|c|}
\hline Parameter & Value \\
\hline$\sigma_{1}$ & 0.971 \\
\hline$\sigma_{2}$ & 3.003 \\
\hline$p_{1}$ & 0.548 \\
\hline$p_{2}$ & 0.452 \\
\hline
\end{tabular}

TABLE 1

Gaussian-Mixture Noise Model Parameters Estimated From Measurements

\section{ERROR BOUND}

\subsection{Derivation}

This section presents a lower bound on estimation error for the linear model (7) under the noise model discussed in Section 2.3. The estimation error vector is defined as $\epsilon=\hat{\mathbf{x}}-\mathbf{x}$, and the error correlation matrix is

$$
\mathbf{R}_{\epsilon}=E\left[\epsilon \epsilon^{T}\right] .
$$

A well-known result in estimation theory known as the MSE, Bayesian or Van Trees bound states that the error correlation matrix is bounded by

$$
\mathbf{R}_{\epsilon} \geq\left(\mathbf{J}_{D}+\mathbf{J}_{P}\right)^{-1}=\mathbf{J}^{-1}
$$

where the inequality indicates that the matrix $\mathbf{R}_{\epsilon}-\mathbf{J}^{-1}$ is positive semi-definite [21]. The matrix

$$
\mathbf{J}_{D}=E\left[\left\{\nabla_{x}[\ln P(\mathbf{y} \mid \mathbf{x})]\right\}\left\{\nabla_{x}[\ln P(\mathbf{y} \mid \mathbf{x})]\right\}^{T}\right]
$$

is known as the Fisher information matrix and represents the information obtained from the data measurements. The matrix

$$
\mathbf{J}_{P}=E\left[\left\{\nabla_{x}[\ln P(\mathbf{x})]\right\}\left\{\nabla_{x}[\ln P(\mathbf{x})]\right\}^{T}\right]
$$

represents the information obtained from a priori knowledge about the random parameters.

We assume that the noise components $\mathbf{n}=$ $\left[n_{1}, \ldots, n_{M}\right]^{T}$ are independent and identically distributed as two-component zero-mean Gaussian mixture random variables as in (10). The noise is independent because we assume nodes are placed at distances larger than the coherence distance of the indoor fading channel.

From (13), we can derive that $\mathbf{J}_{D}$ is given by [22, Eqn 10],

$$
\begin{aligned}
\mathbf{J}_{D} & =\gamma \mathbf{W}^{T} \mathbf{W} \\
\text { where } \gamma & =\int_{-\infty}^{\infty} \frac{\left[f_{n_{i}}^{\prime}(u)\right]^{2}}{f_{n_{i}}(u)} d u
\end{aligned}
$$

and $f_{n_{i}}^{\prime}(u)$ is the derivative of $f_{n_{i}}(u)$ with respect to $u$. When $p_{2}=0$, that is, the distribution of $n_{i}$ is purely Gaussian, $\gamma$ reverts to $1 / \sigma_{1}^{2}$, one over the variance of the distribution. For two-component Gaussian mixtures, we compute $\gamma$ in (15) from numerical integration. For example, for the Gaussian-mixture model parameters calculated from the measurement experiment, as given in Table 1, we find $\gamma=0.548$.

To calculate $\mathbf{J}_{P}$, the prior image distribution $P(\mathbf{x})$ must be known or assumed. One possibility is to assume that $\mathbf{x}$ is a zero-mean Gaussian random field with covariance matrix $\mathbf{C}_{x}$. Then

$$
P(\mathbf{x})=\frac{1}{\sqrt{(2 \pi)^{N}\left|\mathbf{C}_{x}\right|}} e^{-\frac{1}{2}\left(\mathbf{x}^{T} \mathbf{C}_{x}^{-1} \mathbf{x}\right)}
$$




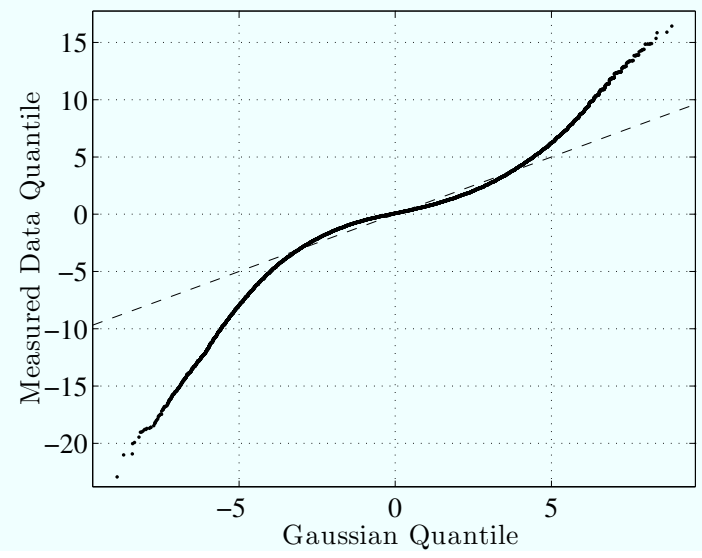

(a) Gaussian Model

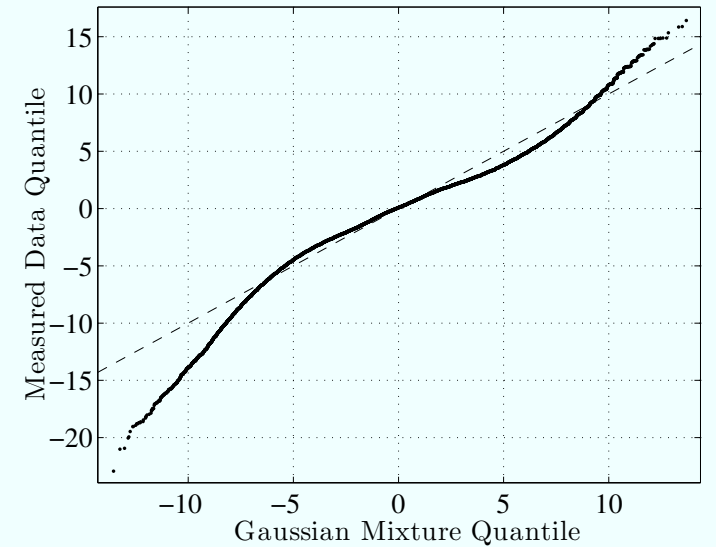

(b) Mixture Model

Fig. 4. Quantile-quantile plots comparing measured RSS data with Gaussian and Mixture distributions.

Plugging (16) into (14) results in

$$
\mathbf{J}_{P}=\mathbf{C}_{x}^{-1} .
$$

These derivations of $\mathbf{J}_{D}$ and $\mathbf{J}_{P}$ lead to the linear MSE bound for RTI

$$
\mathbf{R}_{\epsilon} \geq\left(\gamma \mathbf{W}^{T} \mathbf{W}+\mathbf{C}_{x}^{-1}\right)^{-1} .
$$

An important result of the bound in (18) comes from the following property [21]

$$
E\left[(\mathbf{x}-\hat{\mathbf{x}})_{i}^{2}\right] \geq\left(\gamma \mathbf{W}^{T} \mathbf{W}+\mathbf{C}_{x}^{-1}\right)_{i i}^{-1}=\mathbf{J}_{i i}^{-1}
$$

where $E\left[(\mathbf{x}-\hat{\mathbf{x}})_{i}^{2}\right]$ represents the mean-squared-error for pixel $i$. In other words, the diagonal elements of $J^{-1}$ are the lower bounds on the mean-squared-error for the corresponding pixels.

\subsection{Spatial Covariance Model}

Previous work has shown that an exponential function is useful in approximating the spatial covariance of an attenuation field [23], [16]. The exponential covariance is a close approximation to the covariance that results from modeling the spatial attenuation as a Poisson process, a common assumption for random placement of objects in space. Applying this model, the a priori covariance matrix $\mathbf{C}_{x}$ is generated by

$$
\left[\mathbf{C}_{x}\right]_{k l}=\sigma_{x}^{2} e^{-d_{k l} / \delta_{c}},
$$

where $d_{k l}$ is the distance from pixel $k$ to pixel $l, \delta_{c}$ is a "space constant" correlation parameter, and $\sigma_{x}^{2}$ is the variance at each pixel.

The exponential spatial covariance model is appealing due to its simplicity and low number of parameters. Other models based on different distributions of attenuating objects could also be utilized.

\subsection{Example Error Bounds}

The bound in (19) provides a theoretical basis for determining the accuracy of an image over the network area. The node locations affect which pixels are accurately estimated, and which are not. To visualize how the node locations affect the accuracy of the image estimation, three examples are provided in Fig. 5. Table 2 shows the parameters of the normalized ellipse weighting model that were used to generate these bounds.

\begin{tabular}{|c|c|c|}
\hline Parameter & Value & Description \\
\hline$\Delta_{p}$ & .1 & Pixel width $(\mathrm{m})$ \\
\hline$\lambda$ & .007 & Width of weighting ellipse in (9) $(\mathrm{m})$ \\
\hline$\delta_{c}$ & 1.3 & Pixel correlation constant in $(20)(\mathrm{m})$ \\
\hline$\sigma_{x}^{2}$ & .1 & Pixel variance in $(20)(d B)^{2}$ \\
\hline$\gamma$ & .5483 & Bound parameter in $(19)$ \\
\hline
\end{tabular}

TABLE 2

Reconstruction parameters used to generate MSE bound surfaces shown in Fig 5 .

As seen in the surfaces of Fig. 5, voxels that are crossed by many links have a higher accuracy than voxels that are rarely or never crossed. The voxels in the corners of the square deployment, the sides of the front-back deployment, and the low-density areas in the random deployment, are crossed only by a few links. In some voxels, no links cross at all, and the bound surface is limited only by the covariance of the prior statistics. The known covariance of the image has the effect of smoothing the bound surface, since knowledge of the attenuation of a voxel is statistically related to its neighbors.

\subsection{Effect of Node Density}

The node density plays a key role in the accuracy of an RTI result. Imaging can be expected to be more accurate in areas where nodes are placed closely together than in areas where nodes are spaced at large distances. When 


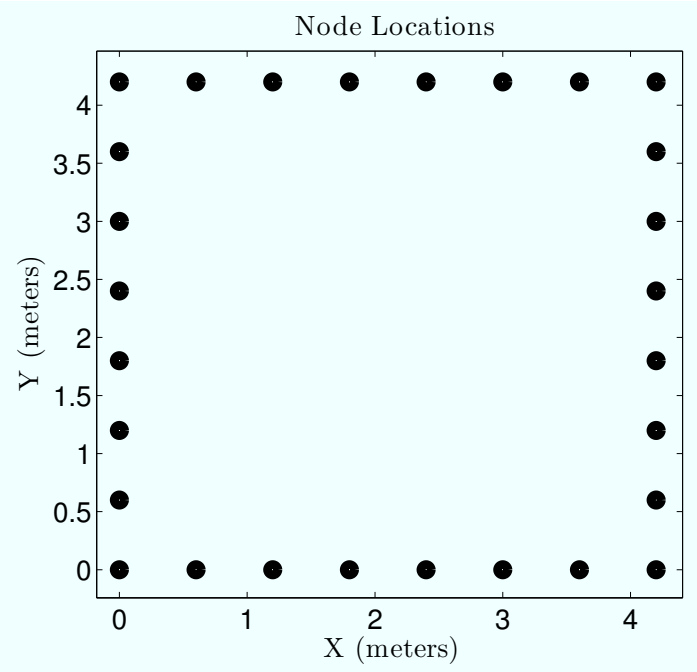

(a) 28 nodes located in a square perimeter, 8 on each side.

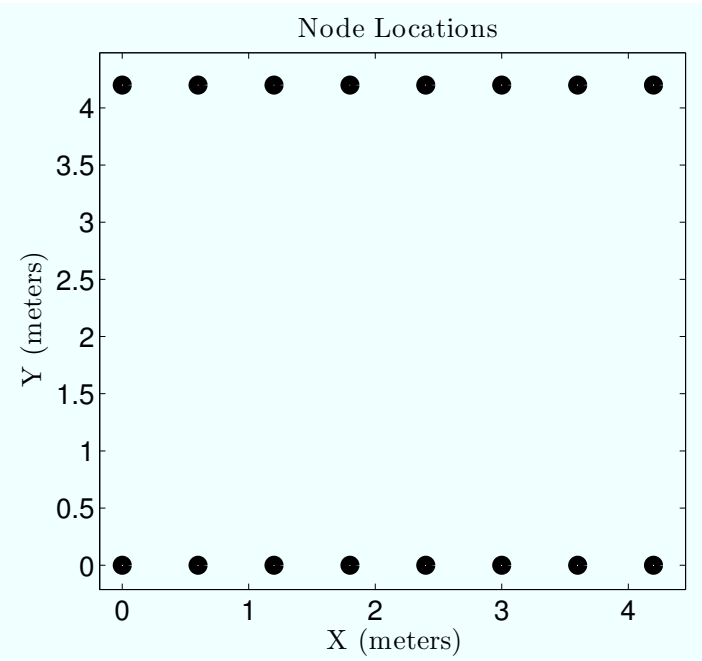

(c) 16 nodes located in a front-back setup, 8 on each side.

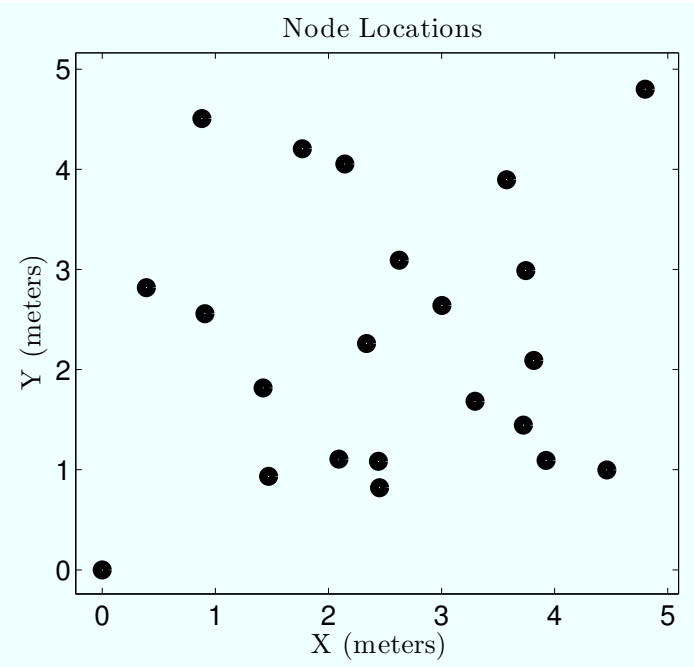

(e) 22 nodes located randomly in a square area.

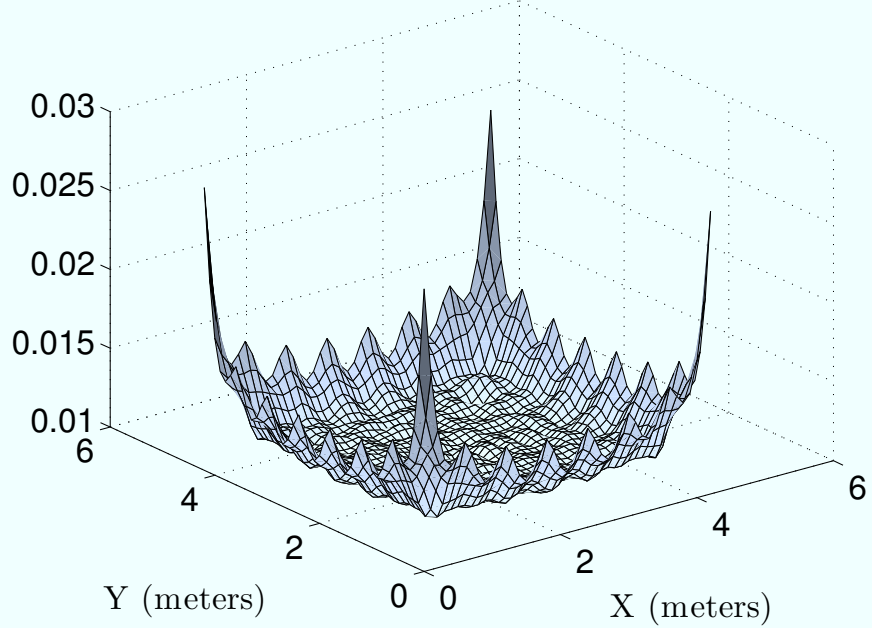

(b) The MSE bound for the node locations shown in Fig. 5(a).

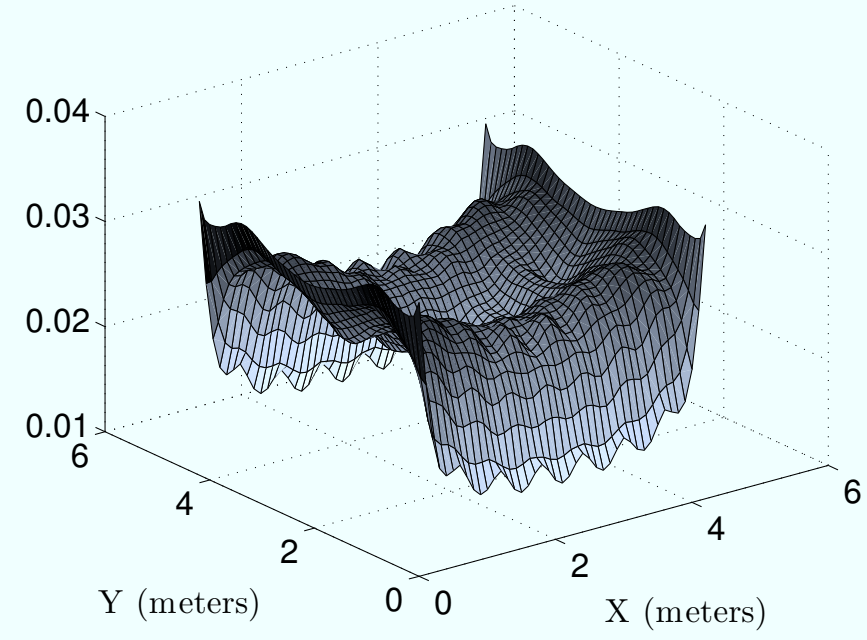

(d) The MSE bound for the node locations shown in Fig. 5(c).

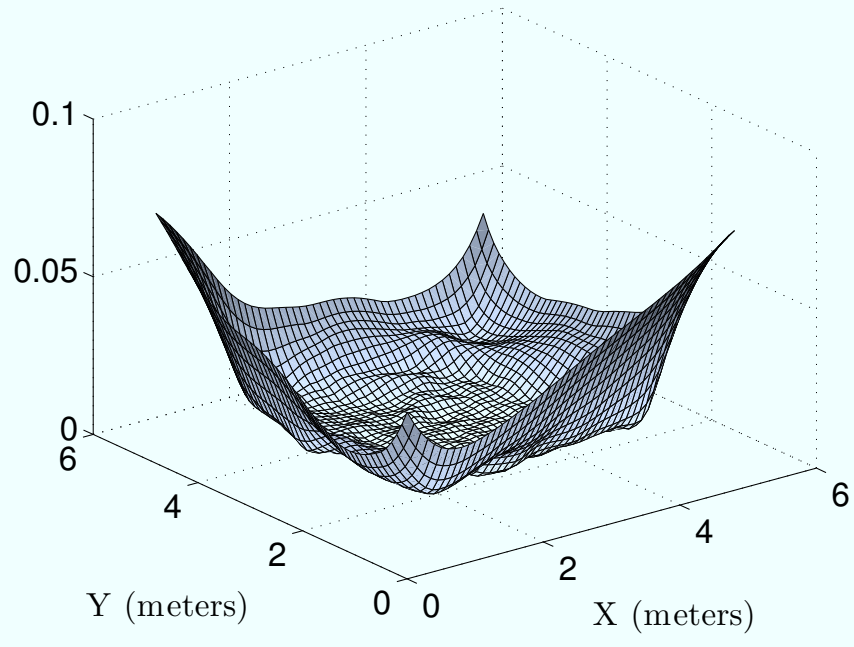

(f) The MSE bound for the node locations shown in Fig. 5(e).

Fig. 5. MSE bound surface plots for a square, front-back, and random node deployments. These plots were generated using the normalized elliptical weight model with a Gaussian image prior. 


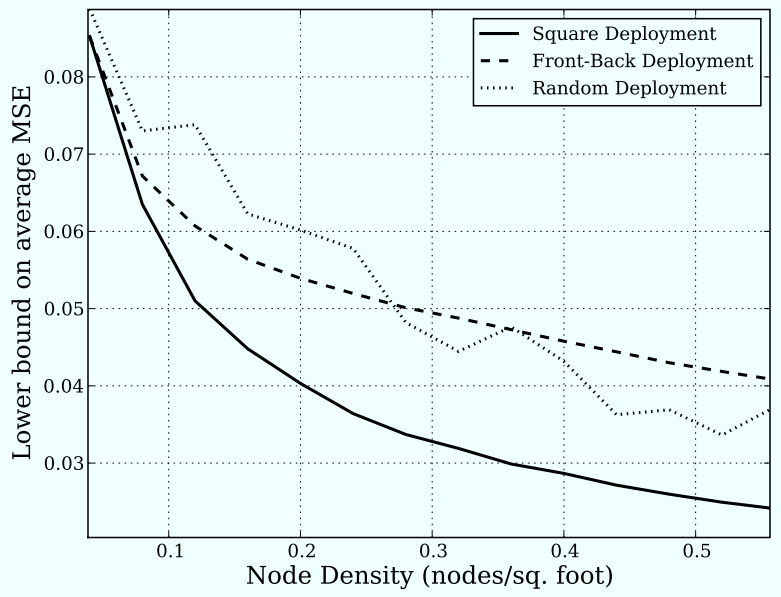

Fig. 6. The lower bound on average MSE vs. node density for three RTI network geometries.

many links pass through a particular area, more RSS information can be used to reconstruct the attenuation occurring in that area. This has the effect of averaging out noise and other corruptions in the measurements. Furthermore, when links are close together, the RSS information is more concentrated on the voxels that are crossed. This is due to the weighting function that is inversely proportional to the square root of the link distance.

To illustrate the effect of node density on the MSE bound, Fig. 6 shows the lower bound on the average MSE over all voxels for the three deployment geometries shown in Fig. 5 as the density is increased. For each point on the curves, the bound surface is calculated, then averaged over all voxels. The parameters are equal to those used previously in Table 2. Each geometry contains the same number of nodes for each point on the curve, and is deployed around the same area. In the square geometry, nodes are placed uniformly around a square area. In the front-back geometry, the same number of nodes are placed along two sides of the square, resulting in the same number of nodes per square foot. In the random geometry, the same number of nodes are randomly placed throughout the square.

In all three cases shown in Fig. 6, the lower bound on average MSE for each deployment decreases rapidly with increasing node density. The square geometry outperforms the others, due to the fact that the entire area of the square is surrounded by nodes. There are very few voxels that are not crossed by at least a few links, and many short links exist that cross the corners of the square. The random geometry performs the worst out of the three when density is low, largely due to the fact that in a random deployment, many voxels will not be crossed by any links. As density increases, the random deployment out-performs the front-back geometry because nodes are closer together, and the density is such that very few areas contain voxels that are not crossed by at least some links.

\section{ImAge Reconstruction}

\subsection{III-posed Inverse Problem}

Linear models for many physical problems, including RTI, take the form of

$$
\mathbf{y}=\mathbf{W x}+\mathbf{n}
$$

where $\mathbf{y} \in \mathbb{R}^{M}$ is measured data, $\mathbf{W} \in \mathbb{R}^{M \times N}$ is a transfer matrix of the model parameters $\mathbf{x} \in \mathbb{R}^{N}$, and $\mathbf{n} \in \mathbb{R}^{M}$ is a measurement noise vector. When estimating an image from measurement data, it is common to search for a solution that is optimal in the least-squared-error sense.

$$
\mathbf{x}_{L S}=\arg \min _{\mathbf{x}}\|\mathbf{W} \mathbf{x}-\mathbf{y}\|_{2}^{2}
$$

In other words, the least-squares solution minimizes the noise energy required to fit the measured data to the model. The least-square solution can be obtained by setting the gradient of (22) equal to zero, resulting in

$$
\mathbf{x}_{L S}=\left(\mathbf{W}^{T} \mathbf{W}\right)^{-1} \mathbf{W}^{T} \mathbf{y}
$$

which is only valid if $\mathbf{W}$ is full-rank. This is not the case in an RTI system.

RTI is an ill-posed inverse problem, meaning that small amounts of noise in measurement data are amplified to the extent that results are meaningless. This is due to very small singular values in the transfer matrix W that cause certain spectral components to grow out of control upon inversion. To see this, $\mathbf{W}$ is replaced by its singular value decomposition (SVD):

$$
\mathbf{W}=\mathbf{U} \Sigma \mathbf{V}^{T}
$$

where $\mathbf{U}$ and $\mathbf{V}$ are unitary matrices, and $\Sigma$ is a diagonal matrix of singular values. Plugging (24) into (23), the least squares solution can be written as

$$
\mathbf{x}_{L S}=\mathbf{V} \Sigma^{-1} \mathbf{U}^{T} \mathbf{y}=\sum_{i=1}^{N} \frac{1}{\sigma_{i}} \mathbf{u}_{i}^{T} \mathbf{y} \mathbf{v}_{i}
$$

where $\mathbf{u}_{i}$ and $\mathbf{v}_{i}$ are the $i$ th columns of $\mathbf{U}$ and $\mathbf{V}$, and $\sigma_{i}$ is the $i$ th diagonal element of $\Sigma$. It is evident that when singular values are zero or close to zero, the corresponding singular basis vectors are unbounded upon inversion.

The heuristic explanation for the ill-posedness of the RTI model lies in the fact that many pixels are estimated from relatively few nodes. There are multiple possible attenuation images that can lead to the same set of measurement data. For example, assume a particular pixel is not crossed by any link in the network. This would result in the same measurement data for every possible attenuation value of that pixel, so inversion of the problem would be impossible.

Regularization involves introducing additional information into the mathematical cost model to handle the ill-posedness. In some methods, a regularization term 
$J(\mathbf{x})$ is added to the minimization objective function of the original problem as

$$
f_{\text {reg }}=f(\mathbf{x})+\alpha J(\mathbf{x}),
$$

where $\alpha$ is the weighting parameter. Small values of $\alpha$ lead to solutions that fit the data, while large values favor the solution that matches prior information.

Some regularization techniques follow from a Bayesian approach, where a certain prior distribution is imposed on the model parameters. Other forms of regularization modify or eliminate small singular values of the transfer matrix. An overview of regularization and image reconstruction in general can be found in [24] and [25].

\subsection{Tikhonov Regularization}

In Tikhonov regularization [24], an energy term is added to the least squares formulation, resulting in the objective function

$$
f(x)=\frac{1}{2}\|\mathbf{W} \mathbf{x}-\mathbf{y}\|^{2}+\alpha\|\mathbf{Q x}\|^{2},
$$

where $\mathbf{Q}$ is the Tikhonov matrix that enforces a solution with certain desired properties.

In this paper, we use a difference matrix approximating the derivative operator as the Tikhonov matrix Q. By minimizing the energy found within the image derivative, noise spikes are suppressed and a smooth image is produced. This form of Tikhonov regularization is known as $H 1$ regularization.

Since the image is two dimensional, the regularization should include the derivatives in both the vertical and horizontal directions. The matrix $\mathbf{D}_{X}$ is the difference operator for the horizontal direction, and $\mathbf{D}_{Y}$ is the difference operator for the vertical direction. The regularized function can be written in this case as

$$
f(\mathbf{x})=\frac{1}{2}\|\mathbf{W} \mathbf{x}-\mathbf{y}\|^{2}+\alpha\left(\left\|\mathbf{D}_{X} \mathbf{x}\right\|^{2}+\left\|\mathbf{D}_{Y} \mathbf{x}\right\|^{2}\right) .
$$

Taking the derivative and setting equal to zero results in the solution

$$
\hat{\mathbf{x}}=\left(\mathbf{W}^{T} \mathbf{W}+\alpha\left(\mathbf{D}_{X}^{T} \mathbf{D}_{X}+\mathbf{D}_{Y}^{T} \mathbf{D}_{Y}\right)\right)^{-1} \mathbf{W}^{T} \mathbf{y} .
$$

One major strength of Tikhonov regularization lies in the fact that the solution is simply a linear transformation $\Pi$ of the measurement data.

$$
\begin{aligned}
\Pi & =\left(\mathbf{W}^{T} \mathbf{W}+\alpha\left(\mathbf{D}_{X}^{T} \mathbf{D}_{X}+\mathbf{D}_{Y}^{T} \mathbf{D}_{Y}\right)\right)^{-1} \mathbf{W}^{T} \\
\hat{\mathbf{x}} & =\Pi \mathbf{y}
\end{aligned}
$$

Since the transformation does not depend on instantaneous measurements, it can be pre-calculated, and then applied for various measurements for fast image reconstruction. This is very appealing for realtime RTI systems that require frequent image updates [15], [26].

The total number of multiplications $N_{\text {mult }}$ required to transform the measurements into the image is the total number of voxels $N$ times the number of unique links $M$ in the network

$$
N_{\text {mult }}=N M=\frac{N\left(K^{2}-K\right)}{2}
$$

where $K$ is the number of nodes in the network. We see that complexity increases linearly as the number of voxels increases, and quadratically as the number of nodes in the network increases.

\section{EXPERIMENTAL Results}

\subsection{Physical Description of Experiment}

A wireless peer-to-peer network containing 28 nodes is deployed for the purpose of testing the capability of RTI to image changed attenuation. Each node is placed three feet apart along the perimeter of a $21 \times 21$ foot square, surrounding a total area of 441 square feet. The network is deployed on a grassy area approximately 15 feet away from the Merrill Engineering Building at the University of Utah. Each radio is placed on a stand at three feet off the ground.

The area surrounded by the nodes contains two trees with a circumference of approximately three feet. The network is intentionally placed around the trees so that static objects exist in the tested RTI system. RTI should only image attenuation that has changed from the time of calibration within the deployment area. Markers are measured and placed in 35 locations within the network so that the humans' locations are known and can be utilized in the subsequent error analysis. A map and photo of the experiment are shown in Fig. 7.

The network is comprised of TelosB wireless nodes made by Crossbow. Each node operates in the $2.4 \mathrm{GHz}$ frequency band, and uses the IEEE 802.15.4 standard for communication. A base station node listens to all network traffic, then feeds the data to a laptop computer via a USB port for the processing of the images. Since the base station node is within range of all nodes, the latency of measurement retrieval to the laptop is low, on the order of a few milliseconds. If a multi-hop RTI network were to be deployed, this latency would certainly increase.

To avoid network transmission collisions, a simple token passing protocol is used. Each node is assigned an ID number and programmed with a known order of transmission. When a node transmits, each node that receives the transmission examines the sender identification number. The receiving nodes check to see if it is their turn to transmit, and if not, they wait for the next node to transmit. If the next node does not transmit, or the packet is corrupted, a timeout causes each receiver to move to the next node in the schedule so that the cycle is not halted.

At the arrival of each packet to the laptop, the RTI program running on the laptop updates a link RSS measurement vector. At each update, the base station hears from only one node in the network, so only RSS values 


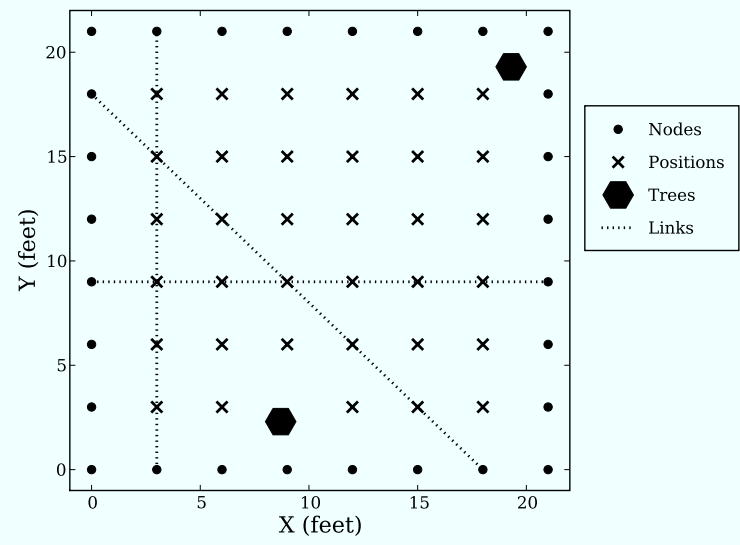

(a) Map

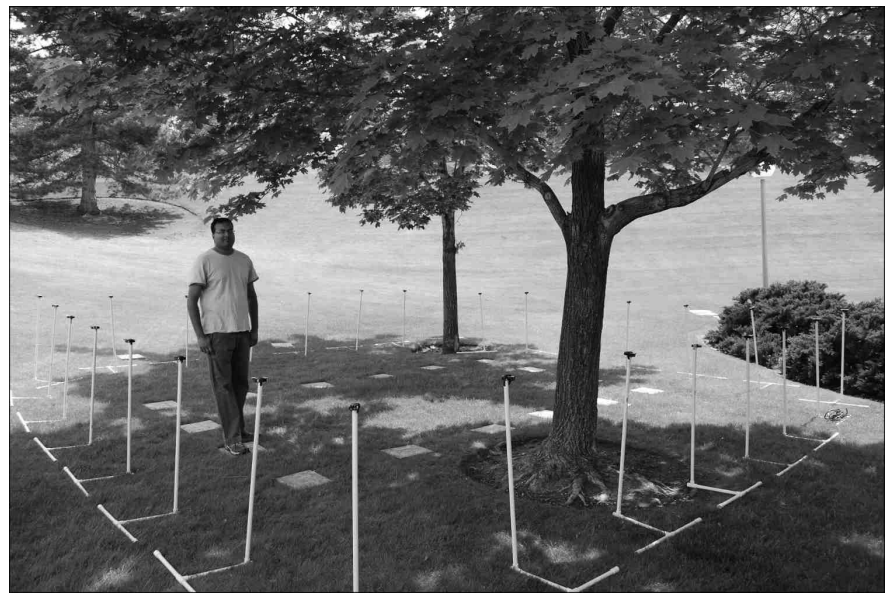

(b) Photo

Fig. 7. The network geometry and links that correspond to Fig. 8 are illustrated in (a). (b) is a photo of the deployed network with an experimenter standing at location $(3,9)$.

on links involving that particular node are updated. Each link's RSS measurement is an average of the two directional links from $i$ to $j$ and $j$ to $i$.

In this experiment, the system is calibrated by taking RSS measurements while the network is vacant from moving objects. The RSS vector is averaged over a 30 second period, which results in approximately 100 RSS samples from each link. The calibration RSS vector provides a baseline against which all other RSS measurements are differenced, as discussed in Section 2. Other methods of calibration could be used in situations where it is impossible to keep the network vacant from moving objects. For example, a single past measurement or a sliding window average of RSS measurement history could be used as the baseline.

\subsection{Effect of Human Obstruction}

Since RTI is based on the assumption that objects shadow individual links in a wireless network, it is helpful to examine the effect of obstructions on a single link. In Fig. 8, a human stands at position $(9,9)$ and RSS measurements for each link are collected. These measurements are compared with the calibration measurements that were taken when the network was vacant.

The top plot in Fig. 8 shows that a significant decrease in RSS, anywhere from 5 to $10 \mathrm{~dB}$, is experienced by link $(0,18)$ to $(18,0)$ as it travels through the obstruction. The middle plot shows that even though the link $(9,0)$ to $(9,21)$ passes through the tree, it still experiences significant loss when the human is present on the LOS path. The bottom plot in the figure shows an example of a link that does not pass through the obstruction, resulting in very little difference in RSS.

In environments where links travel over long distances, or when many objects block the direct LOS path, we expect the effect of a human obstruction to be lessened. In those cases, certain links may experience losses, while others may not. Future research will
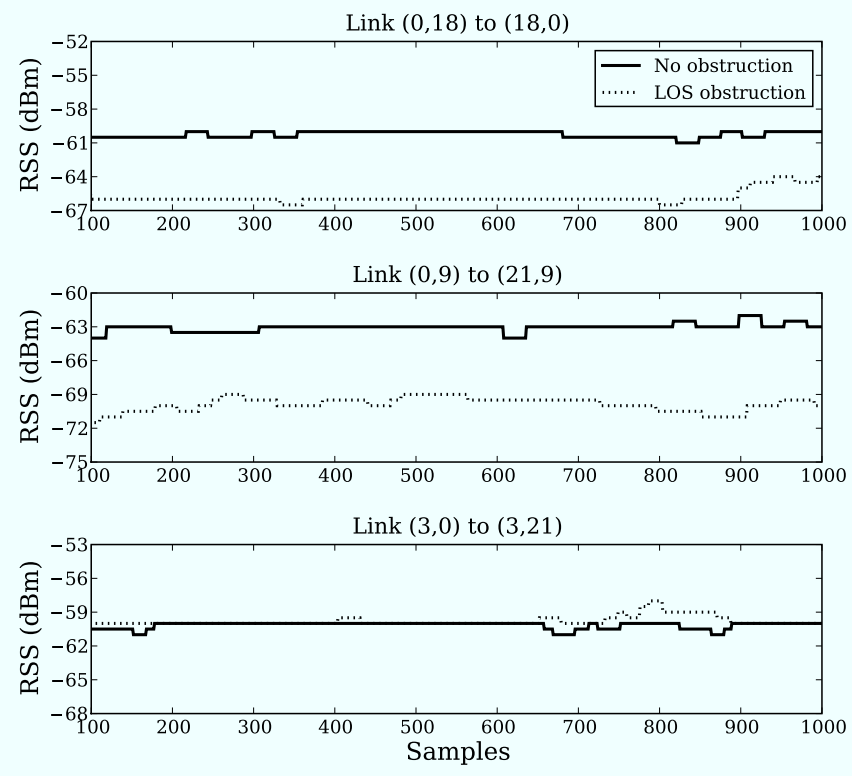

Fig. 8. A comparison of the effect of human obstruction on three links. In the unobstructed case, the network is vacant from human experimenters. In the obstructed case, a human stands at coordinate $(9,9)$.

investigate the effect of human obstruction on a link's RSS when a link passes through walls or other major static obstructions. This will be essential in making the technology practical for the future applications of RTI as previously discussed.

\subsection{Cylindrical Human Model}

To assess the accuracy of RTI images, one must first know or assume the "true" attenuation field that is being estimated. Since imaging the location of humans is the primary goal of RTI, a model for the size, shape, and 


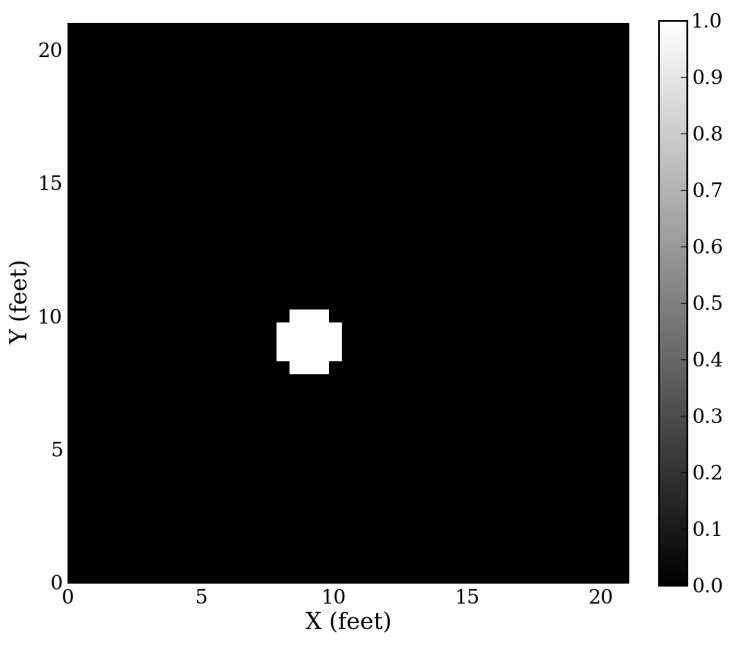

(a) Cylindrical model image

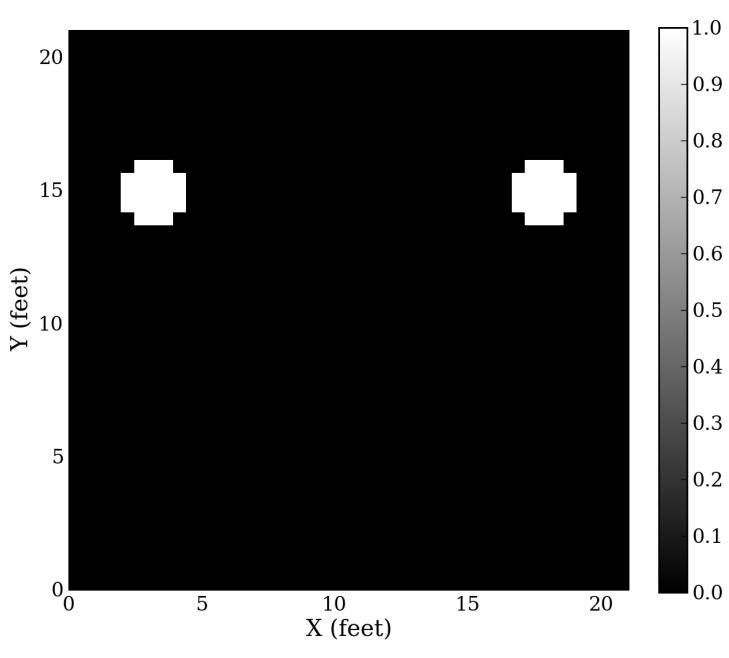

(c) Cylindrical model image

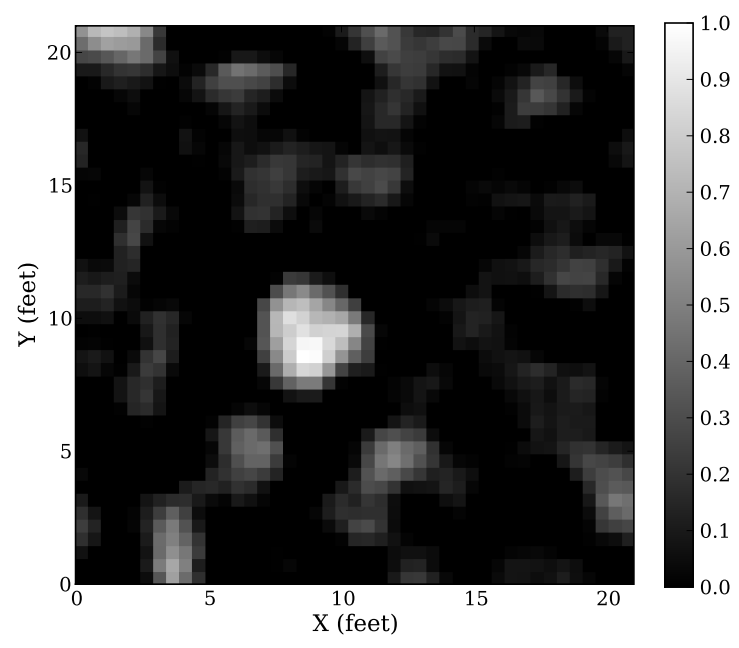

(b) RTI result

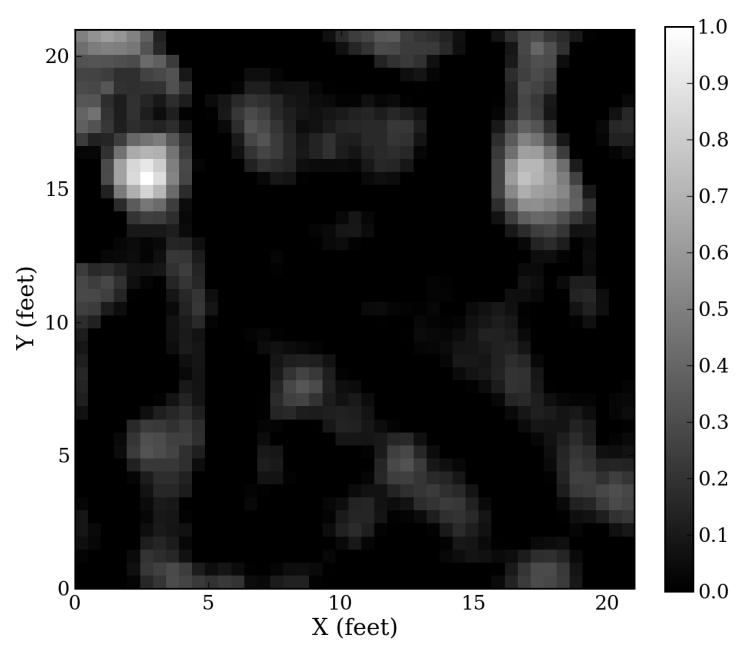

(d) RTI result

Fig. 9. Images of attenuation in a wireless network where each human is modeled as a uniformly attenuating cylinder of radius $R_{H}=1.3$ feet. In (a) and (b), a human stands at coordinate $(9,9)$ and the total squared error is $\epsilon=.021$. In (c) and (d), two humans stand at coordinates $(3,15)$ and $(18,15)$ and the total squared error is $\epsilon=.036$.

attenuation of the human body at the frequencies of interest would be required. This information is difficult to model, since it is dependent on body types, the plane of intersection, and other variables.

For simplicity, a human is modeled as a uniformly attenuating cylinder with radius $R_{H}$. In this case, the "true" image $\mathbf{x}_{c}$ for a human positioned at location $c_{H}$ can be described as

$$
\mathbf{x}_{c j}=\left\{\begin{array}{cc}
1 & \text { if }\left\|\mathbf{x}_{j}-c_{H}\right\|<R_{H} \\
0 & \text { otherwise }
\end{array}\right.
$$

where $\mathbf{x}_{c j}$ is the center location of voxel $j$.

By scaling the image such that the maximum equals one, resulting in the normalized image $\hat{\mathbf{x}}_{N}$, we can define the mean-squared error of the normalized image to be

$$
\epsilon=\frac{\left\|\mathbf{x}_{c}-\hat{\mathbf{x}}_{N}\right\|^{2}}{N}
$$

where $N$ is the number of voxels in the image.

\subsection{Example Images}

Using the model and reconstruction algorithms described in Sections 2 and 4, we present some typical image results for humans standing inside the experimental RTI network. A human stands at coordinate $(9,9)$ and RSS data is measured for a few seconds. The data is averaged for 10 samples per link, and this measurement differenced with the calibration data taken while the network is vacant. Figure 9 displays both the 
"true" attenuation based on the cylindrical model, and the RTI reconstruction using $\mathrm{H} 1$ regularization with the parameters listed in Table 3.

\begin{tabular}{|c|c|c|}
\hline Parameter & Value & Description \\
\hline$\Delta_{p}$ & .5 & Pixel width (feet) \\
\hline$\lambda$ & .01 & Width of weighting ellipse (feet) \\
\hline$\alpha$ & 5 & Regularization parameter \\
\hline$R_{H}$ & 1.3 & Human radius for cylindrical model (feet) \\
\hline
\end{tabular}

TABLE 3

Image reconstruction parameters

Using the cylindrical human model with a radius of $R_{H}=1.3$, the squared error for the single-human image standing at $(9,9)$ was measured to be $\epsilon=.021$. The squared error for the two-person image was measured to be $\epsilon=.036$. These error values are in general agreement with the bounds derived in Section 3.

There are many areas in the images of Fig. 9 where estimated attenuation is above zero, even where no obstruction exists. This is due to the fact that a human not only attenuates a wireless signal, it reflects and scatters it. The simple LOS model used in this paper does not take into account the changes in RSS values due to multipath caused by the obstructions being imaged. For example, a link may be bouncing off the human and destructively interfering with itself on a path that does not cross through the obstruction, thus leading to error in the estimated attenuation. Future research will seek to refine the weighting model used in RTI such that this modeling error is lessened.

\subsection{Effect of Parameters on Image Accuracy}

The weighting and regularization parameters play an important role in generating accurate RTI images. If the problem is regularized too strongly, the resultant images may be too smooth to provide a good indication of obstruction boundaries. If the regularization parameter is set too low, noise may corrupt the results, making it difficult to know if a bright spot is an obstruction or noise.

Another parameter effecting the accuracy of an image is the width of the weighting ellipse. If the ellipse is too wide, the detail of where attenuation is occurring within the network may be obscured. If the ellipse is too narrow, voxels that do in fact attenuate a link's signal may not be captured by the model. This may result in a loss of information that degrades the final image quality.

In this paper, we empirically identify the parameters that provide the most accurate images using the cylindrical human model. For each parameter, images are formed from data measured while a human is standing at one of the known positions, as indicated in Fig. 7 (a). Such an image is formed for each of the possible human positions shown in Fig. 7(a). The squared error is calculated for each image, and averaged over the entire set. This is performed for a varying regularization parameter, while the weighting ellipse parameter is held constant at $\lambda=.1$. Then, it is repeated for varying ellipse parameters while holding the regularization constant at $\alpha=4.5$. The resultant error curves are shown in Fig. 10 .

The curves shown in Fig. 10 show that the choice of regularization and weighting parameters is important in obtaining accurate images. Future research possibly will explore the automatic calculation and adjustment of these parameters. It should be noted that the error curves and optimal values presented are dependent upon the pixel size used in generating the images. The general shape of the curves, however, is similar for different pixel sizes. In this study, pixel size is held constant at .5 feet for all experiments.
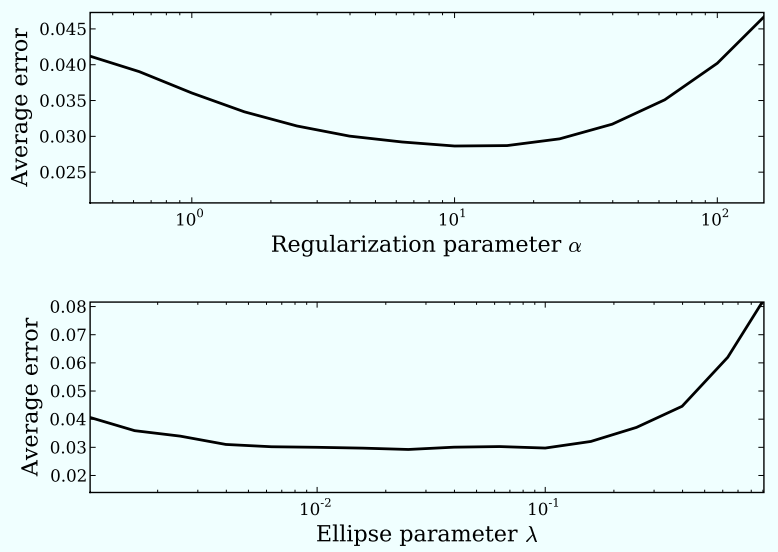

Fig. 10. Error vs. parameter curves. In the first plot, the weighting ellipse width parameter is held constant at $\lambda=$ .1 while the regularization parameter $\alpha$ is varied. In the second, $\alpha=4.5$ and the width of the weighting ellipse is varied.

\section{Conclusion}

Radio tomographic imaging is a new and exciting method for imaging the attenuation of physical objects with wireless networks operating at RF wavelengths. This paper discusses a basic model and image reconstruction technique that has low computational complexity. Experimental results show that RTI is capable of imaging the RF attenuation caused by humans in dense wireless networks with inexpensive and standard hardware.

Future research will be important to make RTI realistic in security, rescue, military, and other commercial applications. First, new models and experiments must be developed for through-wall imaging. In this case, the shadowing and fading caused by many objects in the environment may cause the LOS weighting model to be inaccurate. New and possibly adaptive weighting models will need to be investigated and tested.

Wireless protocols, customized hardware, and signal design are also important for improving RTI. Protocols 
that are capable of delivering low-latency RSS information for large networks will be essential when deploying the technology over large areas. Antennas that direct the $\mathrm{RF}$ energy through an area may reduce the effects of multipath and increase the effect of human presence on signal strength. Custom signals, perhaps taking advantage of frequency diversity may improve the quality of RTI results.

Radio tomographic imaging may provide a low-cost and flexible alternative to existing technologies like ultra-wideband radar. This would enable many applications in the areas of security, search and rescue, police/military, and others.

\section{References}

[1] M. Skolnik, Introduction to Radar Systems. McGraw Hill Book Co., 1980.

[2] A. C. Kak and M. Slaney, Principles of Computerized Tomographic Imaging. The Institute of Electrical and Electronics Engineers, Inc., 1988.

[3] A. Hunt, C. Tillery, and N. Wild, "Through-the-wall surveillance technologies," Corrections Today, vol. 63, July 2001.

[4] D. Estrin, R. Govindan, and J. Heidemann, "Embedding the Internet," Communications of the ACM, vol. 43, pp. 38-41, May 2000.

[5] "Time Domain Corporation." http://www.timedomain.com.

[6] "Cambridge consultants." http://www.cambridgeconsultants.com.

[7] "Camero Tech." http://camero-tech.com.

[8] A. R. Hunt, "Image formation through walls using a distributed radar sensor network," in SPIE Conference on Sensors, and Command, Control, Communications, and Intelligence (C3I) Technologies for Homeland Security and Homeland Defense IV, vol. 5778, pp. 169 174, May 2005

[9] S. L. Coetzee, C. J. Baker, and H. Griffiths, "Narrow band high resolution radar imaging," in IEEE Conf. on Radar, pp. 24-27, April 2006.

[10] H. Griffiths and C. Baker, "Radar imaging for combating terrorism," NATO Advanced Study Institute, http://www. nato-us. org/imaging2006/lecturers. html (Springer, 2006).

[11] M. C. Wicks, B. Himed, L. J. E. Bracken, H. Bascom, and J. Clancy, "Ultra narrow band adaptive tomographic radar," in 1st IEEE Intl. Workshop Computational Advances in Multi-Sensor Adaptive Processing, Dec. 2005.

[12] M. C. Wicks, "RF tomography with application to ground penetrating radar," in 41st Asilomar Conference on Signals, Systems and Computers, pp. 2017-2022, Nov. 2007.

[13] A. M. Haimovich, R. S. Blum, and L. J. C. Jr., "MIMO radar with widely separated antennas," IEEE Signal Processing Magazine, pp. 116-129, January 2008.

[14] M. Youssef, M. Mah, and A. Agrawala, "Challenges: Devicefree passive localization for wireless environments," in MobiCom, (Montreal, Quebec, Canada), pp. 222-229, ACM, September 2007.

[15] N. Patwari and P. Agrawal, "Effects of correlated shadowing: Connectivity, localization, and RF tomography," in IEEE/ACM Int'l Conference on Information Processing in Sensor Networks (IPSN'08), April 2008.

[16] P. Agrawal and N. Patwari, "Correlated link shadow fading in multi-hop wireless networks," IEEE Transactions on Wireless Communications, 2009. (Accepted for Publication).

[17] R. J. Bultitude, "Measurement, characterization, and modeling of indoor 800/900 mhz radio channels for digital communications," IEEE Communications Magazine, vol. 25, June 1987.

[18] R. Ganesh and K. Pahlavan, "Effects of traffic and local movements on multipath characteristics of an indoor radio channel," IEE Electronics Letters, vol. 26, no. 12, pp. 810-812, 1990.

[19] J. Roberts and J. Abeysinghe, "A two-state Rician model for predicting indoor wireless communication performance," in Communications, 1995. ICC '95 Seattle, 'Gateway to Globalization', 1995 IEEE International Conference on, vol. 1, pp. 40-43 vol.1, 1995.
[20] A. P. Dempster, N. M. Laird, and D. B. Rubin, "Maximum likelihood from incomplete data via the EM algorithm," Journal of the Royal Statistical Society, vol. Series B (Methodological) 39, no. 1, pp. 1-38, 1977.

[21] H. L. Van Trees, Detection, Estimation, and Modulation Theory. John Wiley and Sons, 1968.

[22] A. Swami and B. M. Sadler, "On some detection and estimation problems in heavy-tailed noise," Signal Process., vol. 82, no. 12, pp. 1829-1846, 2002.

[23] B. Ripley and W. InterScience, Spatial statistics. Wiley New York, 1981.

[24] C. R. Vogel, Computational Methods for Inverse Problems. SIAM, 2002.

[25] G. Demoment, "Image reconstruction and restoration: Overview of common estimation structures and problems," IEEE Transactions on Acoustics Speech and Signal Processing, vol. 37, December 1989.

[26] J. Wilson and N. Patwari, "Radio tomographic imaging with wireless networks," tech. rep., University of Utah, Sensing and Processing Across Networks Lab, 2008. 